\title{
PI3K Signaling Pathway Regulates the Caspase-9 in Renal Tubular Epithelial Cells
}

\author{
Pei-Fung $\mathrm{Wu} \mathrm{PhD}^{1 *}$ and Cheng-Tse Lee $\mathrm{MD}^{2}$ \\ ${ }^{1}$ Department of Kinesiology, Health and Leisure Studies, National University of Kaohsiung, Kaohsiung 81148, Taiwan \\ ${ }^{2}$ Division of Orthopedics, Zuoying Branch of Kaohsiung Armed Forces General Hospital, Kaohsiung 81342, Taiwan
}

${ }^{\star}$ Corresponding author: Pei-Fung Wu,, Department of Kinesiology, Health and Leisure Studies, National University of Kaohsiung, 700, Kaohsiung University Rd., Nanzih District, Kaohsiung 81148, Taiwan, R.O.C. Fax: +886 7 5919264; Tel: +886 75919212 , E-mail: anniepf@nuk.edu.tw

Received: November 16, 2020; Accepted: November 24, 2020; Published: November 30, 2020

\begin{abstract}
Background: To evaluate how resveratrol regulates the IL-6 signaling in renal cell, pCMV6-IL-6 was overexpressed in the renal tubular epithelial cell line NRK-52E.

Methods: IHC and TUNEL assay were used to identify the localization and apoptosis detection of the overexpression of IL-6 in NRK-52E cells. To identify the effect of overexpressed IL-6, the mitochondrial fraction was isolated and caspase activities and western blotting were performed.

Results: Our results revealed that pCMV6-IL-6 was overexpressed in the nucleus and around the nuclear membrane of the cells. Moreover, the cell membrane showed no IL-6 overexpression, which may be suggest absence of the IL-6/IL-6R binding effect on the cell membrane. Furthermore, the results of the TUNEL assay demonstrated that PCMV6-IL-6-transfected cells showed features of apoptotic cells. The results of the caspase activity assay revealed that resveratrol significantly attenuated IL-6-induced caspase-3 activity but not attenuated IL-6-induced caspase-9 activity, indicating the antiapoptotic ability of resveratrol response on caspase- 3 activity. The PI3K inhibitor could decrease the caspase- 9 level, suggesting that the reduction of the caspase-9 level mediate by through the PI3K signaling pathway.
\end{abstract}

Conclusions: Taken together, our results demonstrated that IL-6 expression not only in the cytosol but also in the nucleus of renal tubular epithelial cells. The PI3K signaling pathway regulates the caspase-9 in renal tubular epithelial cells.

\section{Introduction}

Resveratrol is a naturally occurring stilbene that has been used in anticancer, antiaging, and anti-inflammatory treatment; it has also been applied for cardioprotection and nephroprotection in oxonate-induced hyperuricemic mice and for central nervous system protection [1-3]. Resveratrol provides protection against both acute and chronic kidney injury because of its antioxidant properties and ability to activate sirtuin [4]. Therefore, resveratrol is a useful alternative treatment for renal injury. A previous study suggested that resveratrol acts as an antihyperuricemic and nephroprotective agent in hyperuricemic mice; however, whether resveratrol has beneficial effects on kidney disease in humans and other hyperuricemic animal models remains unclear. Interleukin-6 (IL-6) is a multifunctional cytokine that regulates numerous biological processes including organ development, acute-phase responses, inflammation, and immune responses [5]. The role of IL-6 is not restricted to the immune system; it is also involved in the regulation of metabolic processes. Although IL-6 is a proinflammatory cytokine that promotes inflammation under various pathological conditions involving trans-signaling, its anti-inflammatory and regenerative properties mediated by classic signaling have increasingly been recognized [6-8].The local activation of IL-6 classic and trans-signaling pathways is implicated in renal autoimmune and inflammatory diseases, indicating the importance of IL-6 regulation in renal disease [9]. All kidney resident cells can secrete IL- 6 in certain milieu, but only podocytes express the IL-6 receptor (IL-6R); however, other kidney resident cells do not express IL-6R and use classic IL-6 signaling. Moreover, IL-6 is a well-known activator of signal transducer and activator of transcription 3 (STAT3) [10]. STAT3 has been studied as a transcription factor; a small pool of STAT3 was localized in the mitochondria, where it functioned as a positive regulator of the mitochondrial electron transport chain $[11,12]$. To clarify the regulation of IL-6 in renal cells, recombinant pCMV6-IL-6 was overexpressed in the renal tubular epithelial cell line NRK-52E. Our studies demonstrated that IL-6 expression both in the cytosol and nucleus of renal cells. Resveratrol attenuated IL-6-induced caspase- 3 and caspase- 9 activities. The PI3K inhibitor could decrease the caspase-9 level, suggesting that the PI3K signaling pathway regulates the caspase-9 in renal tubular epithelial cells.

\section{Method}

\section{Preparation of NRK-52E Cells and Transfection}

A normal rat kidney tubular epithelial cell line, NRK-52E (BCRC60086), was purchased from the Food Industry Research and Development Institute, Taiwan. The cells were cultured in Dulbecco's Modified Eagle Medium containing $4.5 \mathrm{~g} / \mathrm{L}$ glucose, $4 \mathrm{mM}$ L-glutamine, and 5\% bovine calf serum (Thermo Fisher Scientific 
Inc., Waltham, MA, USA) and were grown at $37^{\circ} \mathrm{C}$ in a humidified environment with 5\% $\mathrm{CO}_{2}$. pCMV6 and pCMV6-IL-6 cDNA plasmid were purchased from OriGene. The cells were transfected with 2 $\mu \mathrm{g}$ of pCMV6 or pCMV6-IL-6 in each well by using lipofectamine (Thermo Fisher Scientific Inc., Waltham, MA, USA), according to the manufacturer's instructions.

\section{Treatment with Resveratrol}

After NRK-52E cells reached $50-70 \%$ confluence, $10 \mu \mathrm{M}$ of resveratrol (Sigma-Aldrich Corp. MO, USA) were added to the culture medium, and the culture was incubated for $24 \mathrm{~h}$. Control cells were maintained at $37^{\circ} \mathrm{C}$ in a humidified environment with $5 \% \mathrm{CO}_{2}$.

\section{Immunohistochemistry}

The cells were fixed in 10\% phosphate-buffered formalin, blocked with antibody diluent buffer (Dako, Agilent Technologies, Santa Clara, CA, USA), and incubated with anti-DDK antibody (OriGenen Technologies, Inc., Rockville MD, USA) diluted at 1:500 for $60 \mathrm{~min}$ at room temperature. Subsequently, the cells were incubated with secondary antibodies conjugated with horseradish peroxidase (HRP) polymer for 30 $\mathrm{min}$ at room temperature. The cells were then treated with a chromogen, 3,3'-diaminobenzidine tetrahydrochloride (Vector Laboratories, CA, USA), for $10 \mathrm{~min}$. Images were captured using an inverted Nikon ECLIPSE TE2000-S (Nikon Instruments Inc., Melville, NY, USA).

\section{DeadEnd $^{\mathrm{Tm}}$ Colorimetric Apoptosis Detection}

The apoptotic cells were assayed using the terminal deoxynucleotidyl transferase (TdT) dUTP Nick-End Labeling (TUNEL) colorimetric method according to the manufacturer's protocol (Promega Corporation, Madison WI, USA). Briefly, fixed cells were washed, permeabilized, and then incubated with $100 \mu \mathrm{L}$ of $\mathrm{TdT}$ end-labeling cocktail for $60 \mathrm{~min}$ at $37^{\circ} \mathrm{C}$ in a humidified chamber. The cells were blocked with $0.3 \%$ hydrogen peroxide and bound with streptavidin HRP. After washing with PBS, and the cells were incubated with $100 \mu \mathrm{L}$ of 3,3'-diaminobenzidine substrate solution for $10 \mathrm{~min}$ at $25^{\circ} \mathrm{C}$. Images were captured using an inverted Nikon ECLIPSE TE2000-S (Nikon Instruments Inc., Melville, NY, USA).

\section{Caspase-3/CPP32 and Caspase-9 Colorimetric Assay}

Caspase- 3 and caspase- 9 activities were determined using the caspase-3/CPP32 and caspase-9 colorimetric assay kit (BioVision Inc., Milpitas CA, USA), respectively. The cells were washed in cold PBS, resuspended in $50 \mu \mathrm{L}$ cell lysis buffer, and incubated on ice for $10 \mathrm{~min}$. Cell lysates were pelleted, followed by the transfer of supernatants to microcentrifuge tubes. Subsequently, $50 \mu \mathrm{L}$ of cell lysates and $50 \mu \mathrm{L}$ of the reaction buffer was added to microplate wells; $5 \mu \mathrm{L}$ of $4 \mathrm{mM}$ DEVD-pNA substrate for caspase- 3 and $4 \mathrm{mM}$ LEHD-pNA substrate for caspase- 9 were added and then incubated at $37^{\circ} \mathrm{C}$ for $2 \mathrm{~h}$. A control reaction of treated cells without DEVD-pNA or LEHD-pNA was included. The absorbance was measured at $405 \mathrm{~nm}$ using the BioTek Synergy H1 ELISA reader (BioTek Instruments Inc., Winooski VT, USA).

\section{Mitochondrial Fraction Isolation}

The cells were lysed in cytosol extraction buffer containing DTT and protease inhibitors. The samples were maintained on ice for 10 min and then centrifuged at $700 \mathrm{xg}$ for $10 \mathrm{~min}$ at $4^{\circ} \mathrm{C}$. The supernatant was then transferred to a fresh microcentrifuge tube and centrifuged at $10,000 \mathrm{xg}$ for $30 \mathrm{~min}$ at $4^{\circ} \mathrm{C}$. The supernatant was collected as cytosolic fraction, and the pellet was resuspended in mitochondria extraction buffer containing DTT and protease inhibitors as mitochondrial fraction.

\section{SDS-PAGE and Western Blotting}

The protein concentration of supernatants was measured using a BCA kit (Pierce Biotechnology, Inc., USA). For each sample, $10 \mu \mathrm{g}$ or $50 \mu \mathrm{g}$ of the protein lysate was separated on $10 \%$ or $15 \%$ polyacrylamide gels and then transferred to PVDF membranes by using a semidry transfer apparatus (Bio-Rad, Hercules, CA, USA). The membranes were blocked in $5 \%$ nonfat dry milk in TBST buffer $(25 \mathrm{mM}$ of Tris at $\mathrm{pH}$ $7.5,135 \mathrm{mM}$ of $\mathrm{NaCl}$, and $0.15 \%$ Tween-20) for $1 \mathrm{~h}$ and then incubated with anti- $\beta$-Actin (Santa Cruz Biotechnology, Inc.) for $1 \mathrm{~h}$ or with anti-DDK (FLAG) antibody (OriGnen Technologies, Inc., Rockville, MD, USA), anti-p-STAT3-Tyr705 (Santa Cruz Biotechnology, Inc.), or anti-caspase-3, anti-caspase-9, anti-COX IV, anti-p-Akt-Ser473, and anti-p-p38 MAP kinase-Thr180/Tyr182 (Cell Signaling Technology, Inc., Danvers, MA,USA) at $4^{\circ} \mathrm{C}$ for overnight. The blots were washed using TBST and then incubated for 50 min with secondary antibodies conjugated with horseradish peroxidase (Invitrogen, Thermo Fisher Scientific Inc., Waltham, MA, USA). The immunoreactive proteins were detected using an enhanced chemiluminescence detection system (GE Healthcare Bio-Sciences, Marlborough, MA, USA) according to the manufacturer's instructions.

\section{Statistical Analysis}

All data in this study are presented as mean \pm standard error of the mean (SEM) from triplicate measurements. The stained blots were scanned and quantified using ImageJ 1.52a software (NIH, USA). A $p$ value of $<0.05,<0.01$, or $<0.001$ (one-way ANOVA) was considered significant. All statistical analyses were performed using SigmaPlot, Version 13.0 (Systat Software Inc., San Jose, CA, USA).

\section{Results}

\section{IL-6 Overexpression was found in the Nucleus and Around the Nuclear Membrane}

As shown in Figure 1c, pCMV6-IL-6 was overexpressed in the nucleus and around the nuclear membrane of the cells. Moreover, the cell membrane showed no IL- 6 overexpression, which may be suggest absence of the IL-6/IL-6R binding effect on the cell membrane. Besides, IL-6-overexpressing cells showed the presence of apoptotic bodies, as detected using DeadEnd ${ }^{\mathrm{TM}}$ colorimetric apoptosis detection assay (Figure 2d), suggesting that IL-6 regulation may be related to apoptosis.

\section{Resveratrol Attenuated IL-6-induced Caspase-3 Activities in NRK-52E Cells}

As shown in Figure 3a, IL-6 overexpression significantly promoted caspase- 3 activity in NRK-52E cells $(p=0.014)$. Moreover, resveratrol significantly attenuated caspase- 3 activity not only in treatment alone (Figure $2 \mathrm{~b}, p=0.002$ ) but also in pCMV6 transfection cells (Figure 

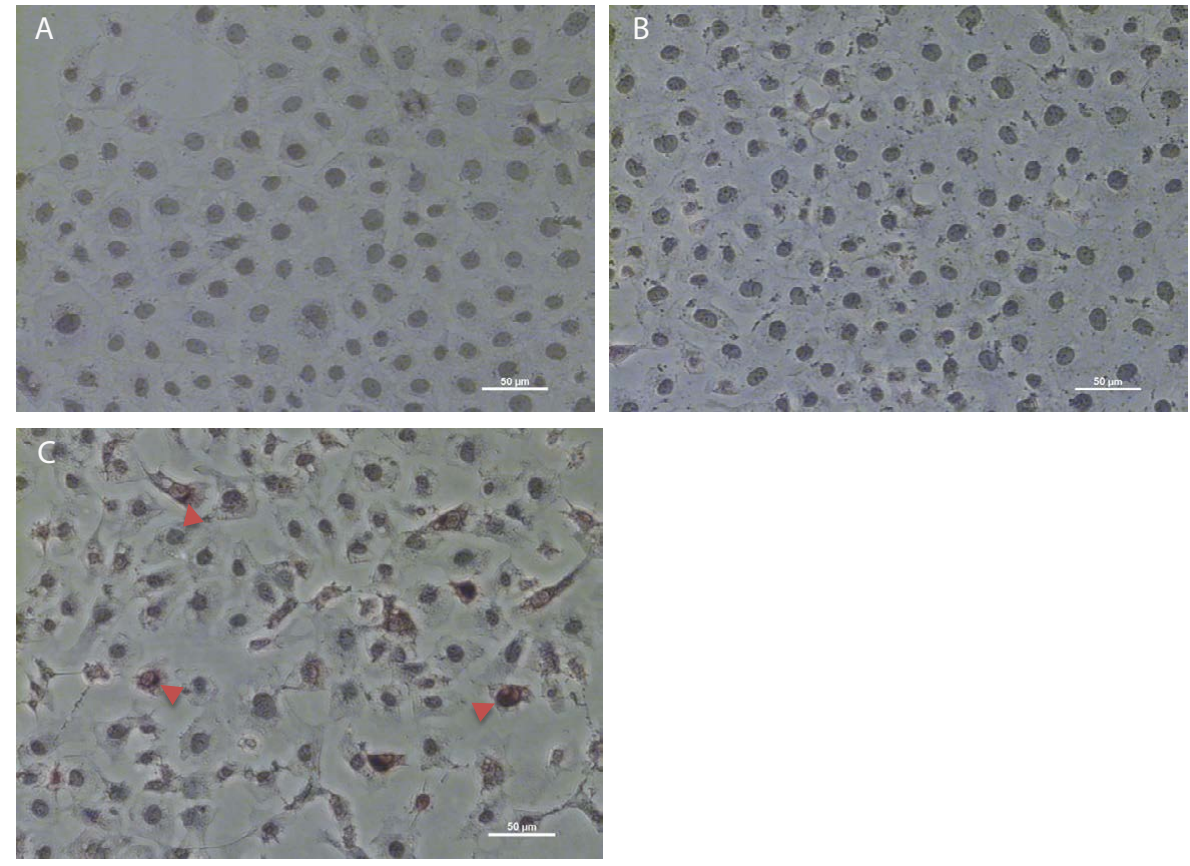

Figure 1: Immunohistochemistry staining of overexpressed IL-6 in NRK-52E cells. (a) The immunohistochemistry staining of the control group. (b) The cells transfected through a pCMV6 vector. (c) The cells transfected through pCMV6-IL-6. The arrow indicates the cell overexpressed IL-6.
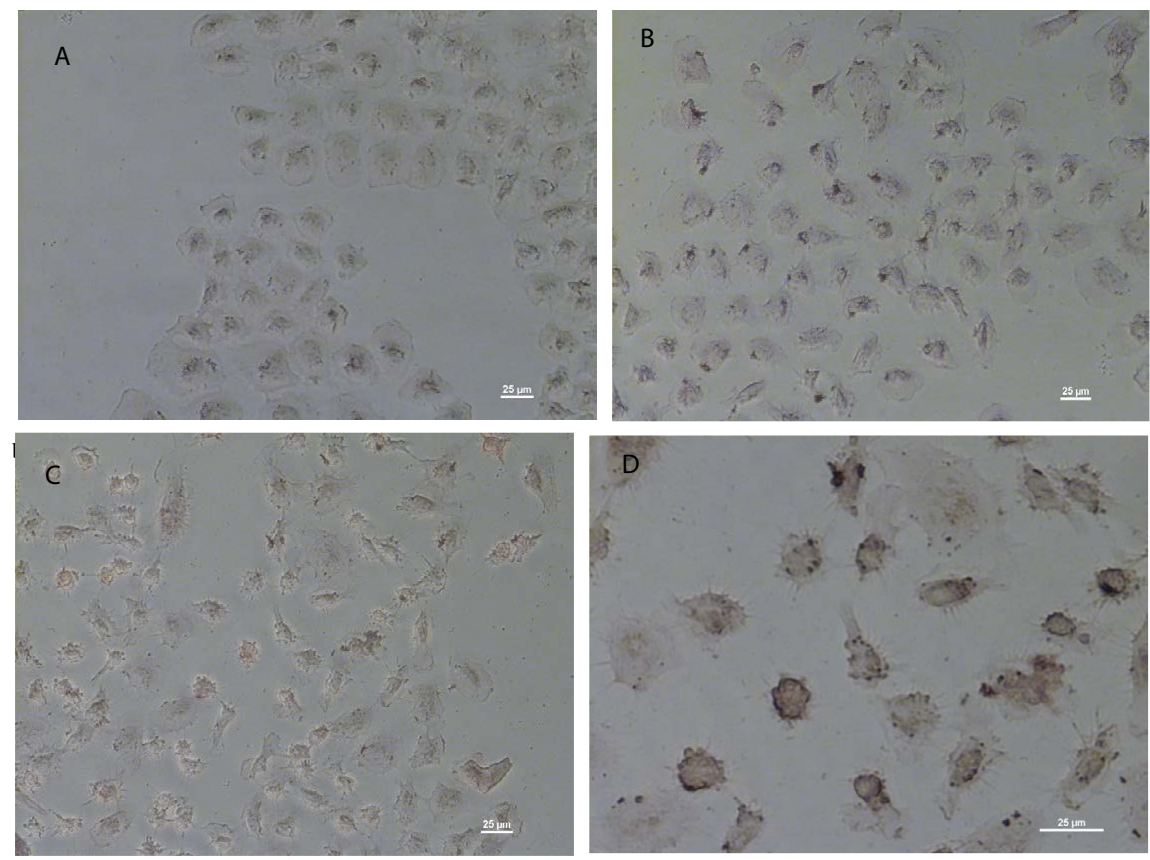

Figure 2: DeadEnd colorimetric apoptosis staining of overexpressed IL-6 in NRK-52E cells. Colorimetric staining of overexpressed IL-6 in NRK-52E cells. (a) The negative control of colorimetric staining. (b) The cells transfected through the pCMV6 vector. (c) Cells transfected through the pCMV6-IL-6 vector. (d) The amplified apoptotic nuclei cells (brown).

$2 \mathrm{~b}, p=0.046$ ) and in pCMV6-IL-6 transfection cells (Figure 2b, $p=$ 0.002). Furthermore, IL-6 overexpression could not regulate caspase- 9 activity and resveratrol also could not regulate overexpressed-IL-6induced caspase-9 activity (Figure 3c).

\section{Resveratrol Attenuated IL-6-induced p-STAT3 Expression}

As shown in Figure 4b, pCMV6-IL-6 expression was found in both cytosol and mitochondrial fractions, and high pCMV6-IL-6 expressed was found in the mitochondrial fraction, indicating the
IL-6 may be involved in the mitochondria regulation of cell function. Moreover, IL-6 overexpression significantly promoted both cytosol and mitochondrial p-STAT3 levels, and resveratrol significantly attenuated the IL-6-induced p-STAT3 level in both cytosol and mitochondrial fractions (Figure $4 \mathrm{c}$ and $4 \mathrm{~d}, p<0.001$ ). Furthermore, IL-6 overexpression increased STAT3 mRNA expression, and resveratrol attenuated IL-6-induced STAT3 mRNA expression (Figure $4 d$ ). Our results showing that IL-6 could activate STAT3 activity in NRK-52E cells. 
A
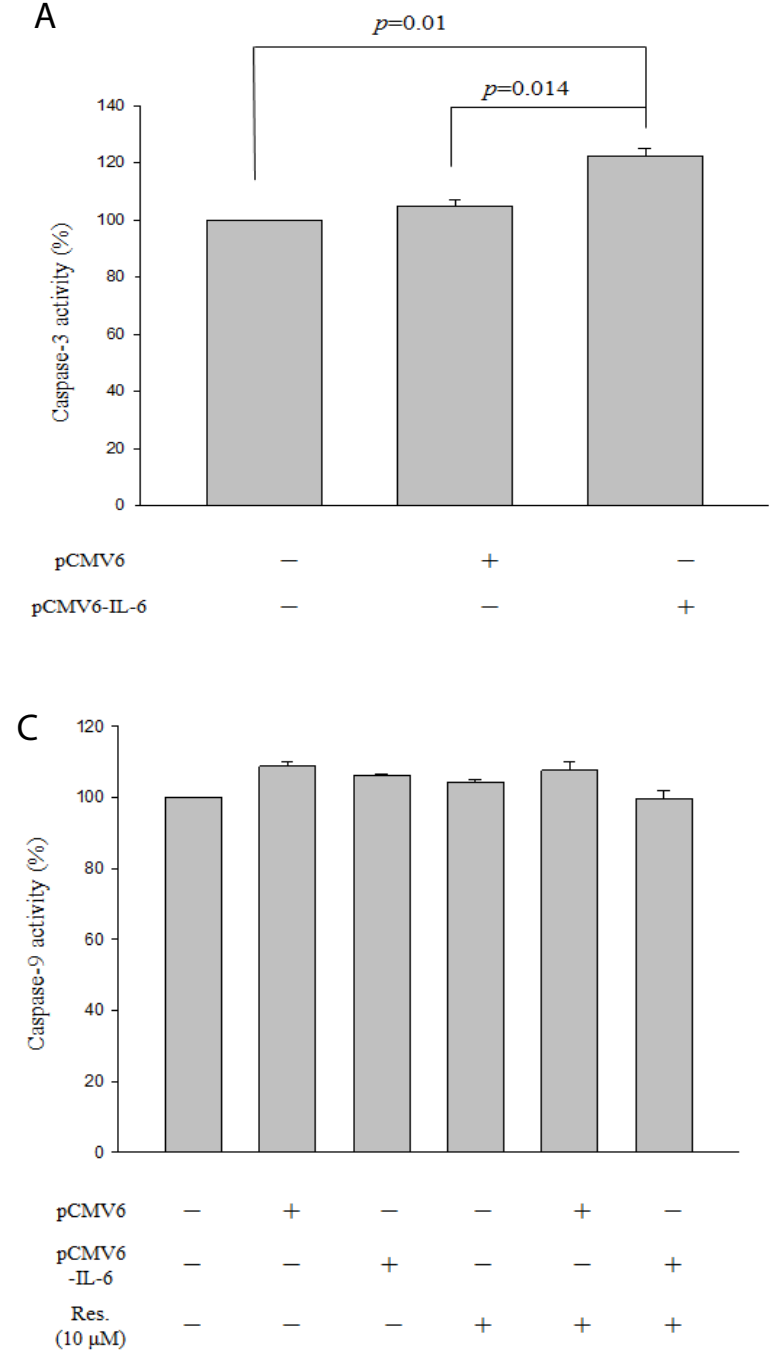

B

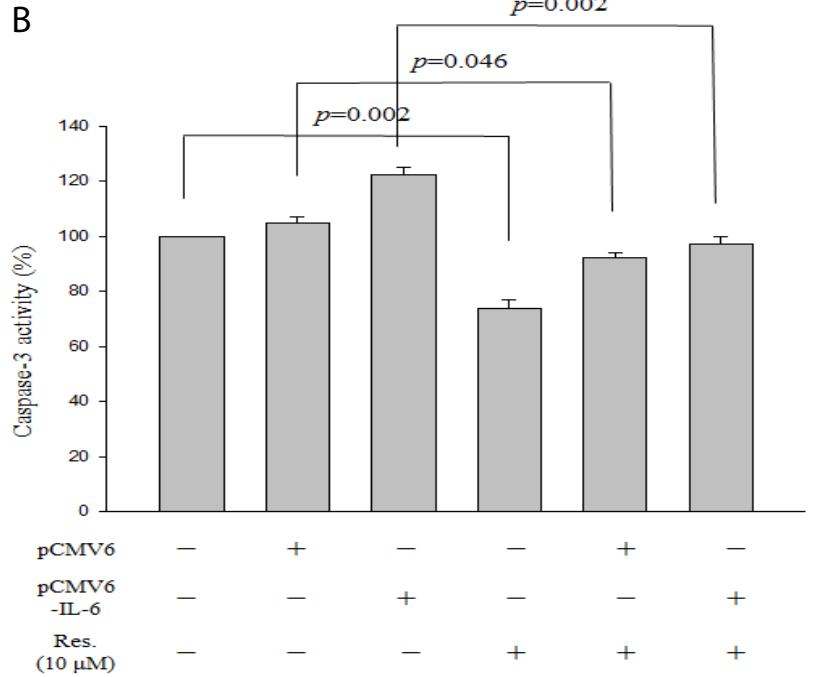

Figure 3: Caspase-3 and caspase-9 activity assays of IL-6 overexpressed NRK-52E cells. (a) Caspase-3 activity of overexpressed IL-6. (b) Caspase-3 activity of overexpressed IL-6 cells containing $10 \mu \mathrm{M}$ resveratrol. (c) Caspase-9 activity of overexpressed IL- 6 cells containing $10 \mu \mathrm{M}$ resveratrol. Data are presented as mean \pm standard error of the mean for the three measurements.

\section{Overexpressed IL-6 Promoted the Caspase-9 Protein Level in Mitochondrial Fraction}

As shown in Figure $4 \mathrm{~b}$ and $4 \mathrm{~g}$, resveratrol significantly promoted the IL-6-induced caspase-9 level only in the mitochondrial fraction (Figure 4g, $p<0.001$ ). Moreover, IL-6 overexpression significantly reduced the caspase- 9 level in the cytosol fraction (Figure 4f, $p<$ 0.001 ) but increased the level in the mitochondrial fraction (Figure $4 \mathrm{~g}, p<0.001)$. Interestingly, resveratrol promoted the IL-6-induced caspase-9 level (Figure 4g, $p<0.001$ ) in the mitochondrial fraction but attenuated IL-6-induced caspase-9 activity in both the cytosol (Figure $3 c, p=0.036$ ) and mitochondria (Figure $4 \mathrm{~h}, p=0.024$ ) fractions, suggesting that resveratrol attenuated IL-6-induced caspase- 9 activity not only in the cytosol fraction but also in the mitochondrial fraction.

\section{Caspase-9 was Mediated by PI3K Signaling Pathway in NRK-52E Cells}

As shown in Figure 5, the caspase-9 level decreased after the PI3K inhibitor LY294002 was added to the culture medium. However, the p38 MAPK inhibitor SB203580 and the STAT3 inhibitor stattic did not influence the caspase-9 level (data not shown), suggesting that the regulation of the caspase- 9 may mediate by through the PI3K signaling pathway. Moreover, LY294002 and SB203580 did not affect the p-STAT3 level or the caspase-3 level, suggesting p-STAT3 and caspase- 3 levels are mediated through other pathways.

\section{Discussion}

Resveratrol affects multiple cellular processes and is an excellent candidate for use in human disorders. Numerous experimental studies and clinical trials have been conducted to analyze the systemic anti-inflammatory, antioxidative, multiorgan protective effects of resveratrol [13-15]. Our previous study explored the resveratrol is a potentially therapeutic strategy for hyperuricemia rats and disclosed immunoreactivity of IL-6 in renal cortex [16]. In this study, to evaluate how resveratrol regulates IL-6 in renal cells, pCMV6IL-6 was overexpressed in the rat tubular epithelial cell line NRK52E. The IL-6 mRNA level in NRK-52E cells was upregulated after treatment with uric acid and was downregulated after treatment with 
A
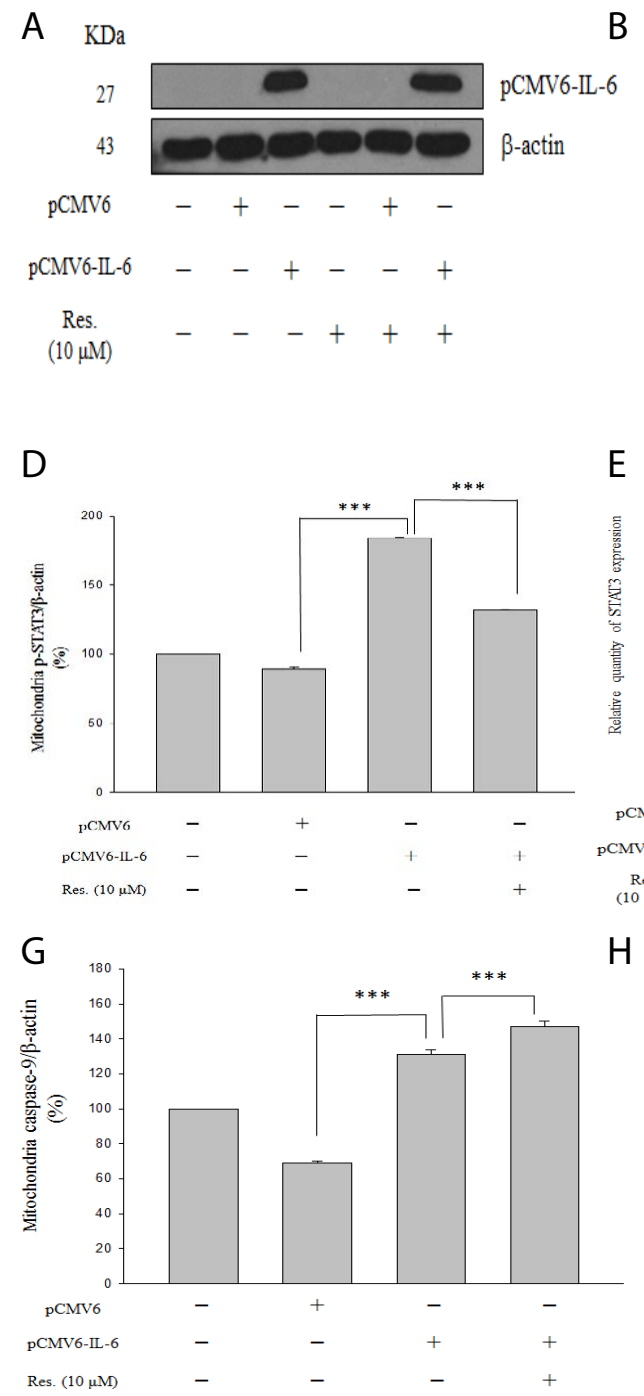

$\mathrm{H}$
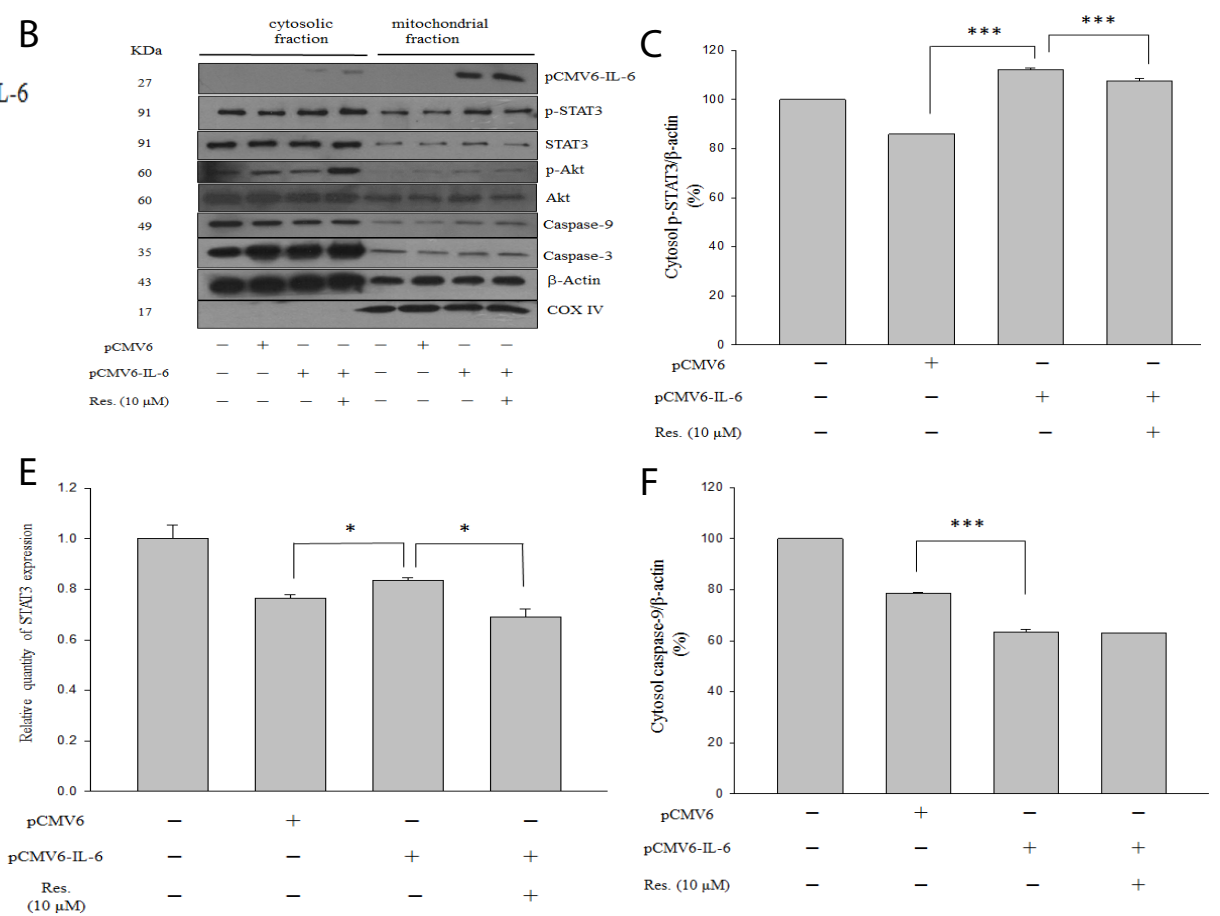

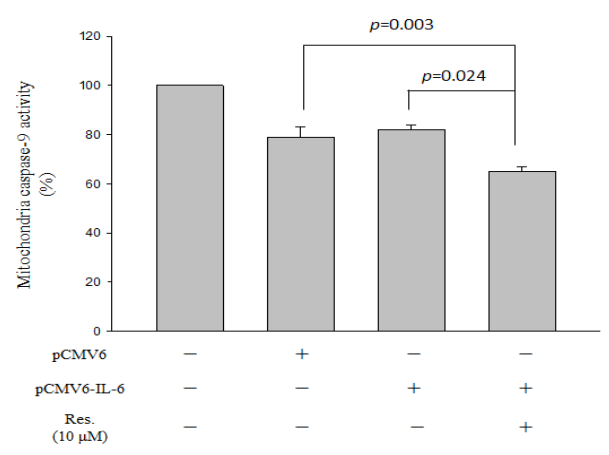

Figure 4: Western blotting analysis of overexpressed IL-6 in cytosol and mitochondrial fractions. Cytosolic fraction corresponds to $50 \mu \mathrm{g}$ of protein lysate prepared. Mitochondrial fraction corresponds to $10 \mu \mathrm{g}$ of protein lysate prepared. (a) Overexpressed IL-6 in with or without resveratrol treatment. (b) Protein level of overexpressed IL-6, p-STAT3, STAT3, p-Akt, Akt, caspase-9, caspase-3, actin and COX IV. (c and f) p-STAT3 and caspase-9 expression in the cytosol, respectively. (d and g) p-STAT3 and caspase-9 expression in the mitochondria, respectively. (e) The mRNA expression of STAT3. (h) Mitochondrial caspase- 9 activity. Anti-IV COX indicates the mitochondrial marker. Data are presented as mean \pm standard error of the mean for the three measurements. ${ }^{*} p<0.05 ;{ }^{* *} p<0.01 ;{ }^{* * *} p<0.001$.

resveratrol, suggesting the anti-inflammatory property of resveratrol (data not shown). It has been demonstrated that the IL-6 exerts proapoptotic effects through the IL- 6 trans-signaling pathway and exerts antiapoptotic effects through the classic pathway $[9,17,18]$. IL-6 signaling through the membrane-bound IL-6R is mostly regenerative and anti-inflammatory, and the signaling of IL-6/sIL-6R has been termed IL-6 trans-signaling, which induces the proinflammatory properties of IL-6. As demonstrated by Nechemia-Arbely et al., IL-6 trans-signaling mediates a protective response to renal injury [19]. In Nechemia-Arbely's study, the administration of an IL-6/sIL-6R fusion protein prevented the onset of acute kidney injury and significantly enhanced survival. Therefore, the role of IL- 6 in the process of cell injury is still controversial. Our study showed that IL- 6 was expressed in the cytosol and nuclear of the renal cells (Figure 1), and low IL-6R mRNA expression was found in the cells (data not shown), indicating the absence of the IL-6/IL-6R binding effect on the cell membrane. Moreover, IL-6-overexpression cells presented apoptotic bodies, as

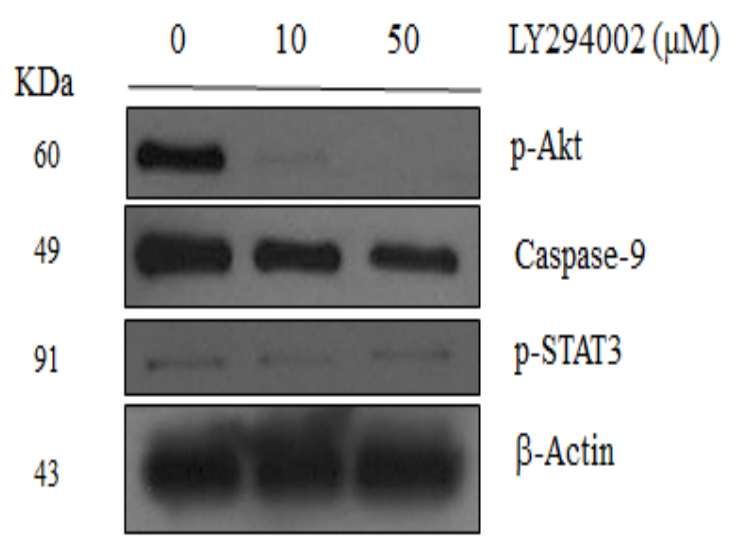

Figure 5: Western blotting analysis of NRK-52E cells treatment with protein inhibitors. Lanes correspond to $50 \mu \mathrm{g}$ of protein lysate prepared. (a) Lysates were analyzed with corresponding antibodies against p-Akt (Ser473), p-P38 (Thr180/Tyr182), caspase-9, p-STAT3 (Tyr705), caspase-3, and $\beta$-Actin. (b) LY294002 containing lysates were analyzed with corresponding antibodies against p-Akt (Ser473), caspase-9, p-STAT3 (Tyr705) and $\beta$-Actin. 
revealed in the DeadEnd ${ }^{\mathrm{TM}}$ colorimetric apoptosis detection assay, suggesting that the regulation of IL-6 may be related to the apoptosis process (Figure 2). Apoptosis is regulated by two interrelated signaling pathways: the extrinsic or death-receptor pathway and the intrinsic or mitochondrial pathway; both pathways use the caspase cascade [20]. Caspase- 9 is a key player in the intrinsic or mitochondrial pathway that is involved in various stimuli, including chemotherapy, stress agent, and radiation [21]. Cytochrome $\mathrm{c}$ is released from the mitochondria to the cytoplasm in cells in response to intrinsic stimuli and forms the apoptosome, which mediates caspase-9 activation [22]. Moreover, caspase- 3 is a major executioner caspase that is cleaved and activated by both caspase- 8 and caspase- 9 initiator caspases [22] In the present study, the results showed that IL- 6 overexpression significantly promoted caspase- 3 activity (Figure 3a) but did not affect caspase- 9 activities (Figure 3c) suggesting the caspase- 3 activity may regulate the overexpressed IL-6-induced apoptosis. However, IL-6 overexpression significantly reduced the cytosolic caspase-9 protein level (Figure 4f, $p<0.001$ ) but significantly promoted the mitochondrial caspase-9 protein level (Figure $4 \mathrm{~g}, p<0.001$ ). The possibility of caspase- 9 shifting from the cytosol to mitochondria, induced by IL- 6 overexpression, remains to be further studied. Moreover, resveratrol only significantly attenuated IL-6-induced caspase- 3 activities (Figure 3b, $p=0.002$ ) but no effect on caspase- 9 activities or overexpressed-IL-6-induced caspase-9 activity (Figure 3c) indicating that resveratrol exerts antiapoptotic ability in attenuated caspase- 3 activities induced by IL- 6 overexpression in NRK-52E cells. STAT3 has been studied as a transcription factor, and a small pool of STAT3 was localized in the mitochondria, where it functioned as a positive regulator of mitochondrial electron transport chain [11-12]. IL-6 is a well-known activator of STAT3 [10]. In the present study, as shown in Figure 4b, pCMV6-IL-6 expression was found in both cytosol and mitochondrial fractions, and pCMV6-IL-6 expression was high in the mitochondrial fraction, indicating that IL- 6 may be involved in the mitochondrial regulation of cell function. As expected, IL-6 overexpression significantly promoted both cytosol and mitochondria p-STAT3 levels (Figure $4 \mathrm{c}$ and $4 \mathrm{~d}, p<0.001$ ), indicating that IL- 6 could activate STAT3 activity in NRK-52E cells. Resveratrol can exert its anticancer effects by negative regulation of STAT3/5 signaling cascade [23]. Our results also showed that resveratrol not only significantly attenuated the IL-6-induced p-STAT3 level in both cytosol and mitochondrial fractions (Figure $4 \mathrm{c}$ and $4 \mathrm{~d}, p<0.001$ ) but also significantly decreased IL-6-induced STAT3 mRNA expression (Figure $4 \mathrm{e}, p<0.05$ ), indicating that resveratrol may downregulate IL-6-induced STAT3 mRNA expression. Human caspase-9 is phosphorylated on Ser196 by Akt/PKB, resulting in the attenuation of its activity, which suggests that the PI3K signaling pathway plays a central role in antiapoptosis [24]. Moreover, the ERK MAPK pathway inhibits caspase-9 activity through direct phosphorylation at Thr 125 [25]. Past studies have suggested that at least one signaling pathway modulates caspase-9. Our study showed that the caspase-9 level was inhibited by the PI3K inhibitor (LY294002) (Figure 5) but could not be inhibited by the MAPK inhibitor (SB203580) or STAT3 inhibitor (stattic) (data not shown), indicating that in NRK-52E cells, the regulation of capase- 9 may mediated by PI3K signaling.
Taken together, our important finding in this study explored: (1) The overexpressed IL- 6 both located in the cytosol and nucleus of renal cells. (2) IL-6 overexpression significantly reduced the caspase-9 level in the cytosol fraction but increased the level in the mitochondrial fraction. (3) The PI3K signaling pathway regulates the caspase-9 in renal tubular epithelial cells. Further research is particularly important on elucidate IL- 6 overexpression how to mediate the reduction of the caspase- 9 level and the possibility of caspase- 9 shifting from the cytosol to mitochondria in response to IL-6.

\section{Abbreviations}

IL-6: Interleukin-6

IL-6R: IL-6 Receptor

STAT3: Signal Transducer and Activator of Transcription 3

p-STAT3: p-Signal Transducer and Activator of Transcription 3.

\section{Acknowledgement}

This study was funded by grant from Ministry of Science and Technology, Taiwan (MOST 106-2320-B-390-001) and Zuoying Branch of Kaohsiung Armed Forces General Hospital, Kaohsiung, Taiwan (ZBH106-08).

\section{Conflict of Interest Statement}

The authors have declared no conflict of interest.

\section{Funding}

This study was funded by grant from Ministry of Science and Technology, Taiwan (MOST 106-2320-B-390-001) and Zuoying Branch of Kaohsiung Armed Forces General Hospital, Kaohsiung, Taiwan (ZBH106-08).

\section{Author Contributions}

$\mathrm{Wu}$, P.F. and Lee, C.T. conceived, designed and performed the experiments. $\mathrm{Wu}, \mathrm{P}$.F. analyzed the data and wrote the paper.

\section{References}

1. Alkhalaf M, El-Mowafy A, Renno W, Rachid O, Ali A, et al. (2008) Resveratrolinduced apoptosis in human breast cancer cells is mediated primarily through the caspase-3-dependent pathway. Arch Med Res 39: 162-168. [crossref]

2. Kovacic P, Somanathan R (2010) Multifaceted approach to resveratrol bioactivity: Focus on antioxidant action, cell signaling and safety. Oxid Med Cell Longev 3: 86100. [crossref]

3. Meng Y, Ma QY, Kou XP, Xu J (2005) Effect of resveratrol on activation of nuclear factor kappa-B and inflammatory factors in rat model of acute pancreatitis. World $J$ Gastroenterol 11: 525-528.

4. Malhotra A, Bath S, Elbarbry F (2015) An Organ System Approach to Explore the Antioxidative, Anti-Inflammatory, and Cytoprotective Actions of Resveratrol. Oxid Med Cell Longev

5. Scheller J, Chalaris A, Schmidt-Arras D, Rose-John S (2011) The pro- and antiinflammatory properties of the cytokine interleukin-6. Biochim Biophys Acta 1813: 878-888. [crossref]

6. Akeson G, Malemud CJA (2017) Role for Soluble IL-6 Receptor in Osteoarthritis. J Funct Morphol Kinesiol 2. [crossref]

7. Hirano T (1998) Interleukin 6 and its receptor: ten years later Int Rev Immunol 16 : 249-284. [crossref] 
8. Rose-John S (2012) IL-6 trans-signaling via the soluble IL-6 receptor: importance for the pro-inflammatory activities of IL-6. Int J Biol Sci 8:1237-1247. [crossref]

9. Su H, Lei CT, Zhang C (2017) Interleukin-6 Signaling Pathway and Its Role in Kidney Disease: An Update. Front Immunol 8. [crossref]

10. Heinrich PC, Behrmann I, Müller-Newen G, Schaper F, Graeve L (1998) Interleukin6-type cytokine signalling through the gp130/Jak/STAT pathway. Biochem J 334: 297 314. [crossref]

11. Wegrzyn J, Potla R, Chwae YJ, Sepuri N.B, Zhang Q, et al.(2009) Function of mitochondrial Stat3 in cellular respiration. Science 323: 793-797. [crossref]

12. Yang R, Rincon M (2016) Mitochondrial stat3, the need for design thinking. Int J Biol Sci; 12: 532-544. [crossref]

13. Carter LG, D’Orazio JA, Pearson KJ (2014) Resveratrol and cancer: focus on in vivo evidence. Endocr Relat Cancer 21. [crossref]

14. Xia N, Daiber A, Förstermann U, Li H (2017) Antioxidant effects of resveratrol in the cardiovascular system. Br J Pharmacol 174: 1633-1646. [crossref]

15. Vervandier-Fasseur D, Latruffe N (2019) The Potential Use of Resveratrol for Cancer Prevention. Molecules 24. [crossref]

16. Lee CT, Chang LC, Liu CW, Wu PF (2017) Negative correlation between serum uric acid and kidney URAT1 mRNA expression caused by resveratrol in rats. Mol Nutr Food Res 61. [crossref]
17. Rose-John S, Waetzig GH, Scheller J, Grötzinger J, Seegert D (2007) The IL-6/sIL-6R complex as a novel target for therapeutic approaches. Expert Opin Ther Targets 11: 613-624. [crossref]

18. Rothaug M, Becker-Pauly C, Rose-John S (2016) The role of interleukin-6 signaling in nervous tissue. Biochim Biophys Acta 1863(6 Pt A): 1218-1227. [crossref]

19. Nechemia-Arbely Y, Barkan D, Pizov G, Shriki A, Rose-John S, et al. (2008) Axelrod JH. IL-6/IL-6R axis plays a critical role in acute kidney injury. J Am Soc Nephrol 19: 1106-1115. [crossref]

20. Salvesen GS (2002) Caspases: opening the boxes and interpreting the arrows. Cell Death Differ 9: 3-5. [crossref]

21. Li P, Zhou L, Zhao T, Liu X, Zhang P, et al. (2017) Caspase-9: structure, mechanisms and clinical application. Oncotarge 8: 23996-24008. [crossref]

22. Jin Z, El-Deiry WS (2005) Overview of cell death signaling pathways. Cancer Bio Ther 4: 139-163.

23. Kim C, Baek SH, Um JY, Shim BS, Ahn KS (2016) Resveratrol attenuates constitutive STAT3 and STAT5 activation through induction of PTPE and SHP-2 tyrosine phosphatases and potentiates sorafenib-induced apoptosis in renal cell carcinoma. BMC Nephrol 25: 17. [crossref]

24. Cardone MH, Roy N, Stennicke HR, Salvesen GS, Franke TF, Stanbridge E, Frisch S, Reed JC (1998) Regulation of cell death protease caspase-9 by phosphorylation. Science 282: 1318-1321. [crossref]

25. Allan LA, Morrice N, Brady S, Magee G, Pathak S, et al. (2003) Inhibition of caspase- 9 through phosphorylation at Thr 125 by ERK MAPK. Nat Cell Biol 5: 647654. [crossref]

\section{Citation:}

Pei-Fung Wu, Cheng-Tse Lee (2020) PI3K Signaling Pathway Regulates the Caspase-9 in Renal Tubular Epithelial Cells. J Pharmacol Pharm Res Volume 3(3): 1-7. 